\title{
EXPERIMENTAL STUDY ON BEHAVIOR OF TURBULENCE IN A FULLY DEVELOPED PIPE FLOW
}

\author{
Ryuzo ITO, Yushi HIRATA, Osamu KITA, Sadahiro SENO \\ KAZUO OMODAKA AND RYOICHI FUKUI \\ Department of Chemical Engineering, Osaka University, \\ Toyonaka 560
}

\begin{abstract}
To investigate the Lagrangian behavior of turbulent eddies in fully turbulent pipe flow, two point space-time correlations were measured by the electrochemical method; $s-s$ correlation between the wall shear stresses spaced along the axial direction, $s-u$ correlation between the wall shear stress and the Iocal axial velocity in the same cross section, and $u-u$ correlation between the local axial velocities in the same cross section.

The correlation for time lag, defined as the delay time at which the correlation function becomes maximum, has been obtained for each cross correlation.

The axial distribution of the time lags in the s-s correlation demonstrates that the axial velocity of turbulent eddies near the wall is 0.455 times the average velocity but is not proportional to the friction velocity as reported by $P y$.

A model on transmission of turbulent disturbance is proposed here upon which the transmission velocities in the radial direction have been obtained from the $s-u$ and $u$ - $u$ correlations respectively. The transmission velocity from the $s-u$ correlation is proportional to $(y / R)^{0.2}$, which corresponds to the radial velocity of an inrushing eddy. On the other hand, the transmission velocity in the bulk region obtained from the $u-u$ correlation decreases towards the center.
\end{abstract}

\section{Introduction}

A complete theory of the phenomena of turbulent diffusion is not available even in the simplest case of homogeneous isotropic turbulence. Some experimental studies on dispersion of scalar quantities, such as temperature or concentration, in turbulent flows have been carried out by Corrsin ${ }^{11}$ and others. In spite of its importance, little is known about the behavior of turbulent eddies themselves.

To determine the spatial variations of the turbulent structure and the statistical character of the turbulent convected frame, a two point space-time correlation technique has been utilized in a turbulent boundary layer by Blackwelder and Kovasnay ${ }^{1)}$ and Favre et $a l .^{4}$, in a two-stream mixing layer by Jones et $a l^{5}{ }^{5}$, and in pipe flow by the authors below.

This technique is applicable to measurements of the longitudinal velocity of disturbance near the wall with imbedded two point detectors spaced along the mean flow direction. Show and Hanratty ${ }^{10)}$ measured electrochemically the local mass transfer rate at the wall and estimated the convective velocity of dis-

Received October 1,1976. Correspondence concerning this article should be addressed to Y. Hirata. O. Kita is now with Hitachi Ltd., Kasado Works, Kudamatsu 744, S. Seno with Fuji Photo Film Co. Ltd., Minami-Asigara 250-01, K. Omodaka with Kao Soap Co. Ltd., Wakayama 641, and R. Fukui with Toyo Engineering Co., Funabashi 273. turbance moving downstream $\left.U_{s}^{+} \sim 1 . \mathrm{Liu}^{8}\right)$ used the streaming potential measurements in a turbulent pipe flow and obtained a disturbance velocity of about 0.7 to 0.8 times the bulk average velocity. $\mathrm{Py}^{9)}$ used the electrochemical wall shear stress meters and found that the disturbance velocities $U_{s}$ depended on the distance between two point electrodes and seem to approach a constant, $U_{s}^{+} \sim 6.3$, as the distance decreases.

On the other hand, Eckelmann ${ }^{3}$ measured the space-time correlation between fluctuations of velocity gradient at the wall and those of local velocity at $y^{+}$ apart from the wall. He found that the correlation had very high values in the range of $y^{+} \leq 5$ while having some time lags. Based on the simple penetration model, the transmission velocity towards the wall was estimated as $V_{s}^{+}=1$ for $y^{+} \leq 5$. This correlation gives a better understanding to the turbulent structure near the wall, together with the visual investigations by Kim et al. ${ }^{6)}$ and Corino and Brodkey ${ }^{2}$ who found intermittent bursting and inrushing phenomena.

The present work is an effort to clarify the statistical behavior of turbulence by applying two point spacetime correlation technique to the velocity and shear stress fluctuations in a fully developed turbulent pipe flow; also, a transmission model of turbulent disturbance will be proposed, upon which the transmis- 
sion velocity is obtained.

\section{Experimental Equipment and Techniques}

The investigation was conducted in a straight plastic pipe of $67 \mathrm{~mm}$ diameter and the test section had an $L / D=60$, where the flow was considered as being fully developed for $R e>10^{4}$.

For measurements of fluid velocity and wall shear stress, the diffusion controlled redox reaction of ferri-ferro cyanide ions was utilized.

Twelve point electrodes, $0.3 \mathrm{~mm} \phi \mathrm{Pt}$, imbedded at the wall of the test section for measurement of the cross correlation between local wall shear stresses were arranged spaced along a line parallel to the pipe axis, the separation of which were $0.134,0.273,0.457$, $0.758,0.985,1.266,1.640,2.046,2.560,3.385,4.385 \mathrm{~cm}$ (center to center).

For measurement of fluid velocity, a blunt-nose type electrode, $0.2 \mathrm{~mm} \phi \mathrm{Pt}$, was used.

For the cross correlations between fluctuations of wall shear stress, $s$, and velocity, $u$, at a distance $y$ from the wall, a blunt-nose electrode was traversed in the radial direction at the same cross section with the wall shear stress meter as shown in Fig. 1. For the measurement of $s-u$ correlations, the range of $R e$ : $38200,76400,114600$.

For the cross correlations of two velocity fluctuations at $y_{r}$ and $y_{r}+\Delta y$, the reference electrode was fixed at $y_{r}$ and the other was traversed further away from the wall than the reference one. The measurements were carried out at $y_{r}$ of $3.92,7.27,10.65$, $13.74,17.35 \mathrm{~mm}$ and the range of $\Delta y$ to be 0.89 $15.7 \mathrm{~mm}$ at $R e$ of 89600 only.

The liquid used was a solution of $0.01 \mathrm{~mol} / l$ $\mathrm{K}_{4} \mathrm{Fe}(\mathrm{CN})_{6}$ and $\mathrm{K}_{3} \mathrm{Fe}(\mathrm{CN})_{6}$, and $1 \mathrm{~mol} / l \mathrm{NaOH}$ as a supporting electrolyte, and held at a temperature of $30^{\circ} \pm 0.02^{\circ} \mathrm{C}$. The properties of the solution: $\rho=$ $1.038 \mathrm{~g} / \mathrm{cm}^{3}, \mu=0.905 \mathrm{cp}$.

The experimental data were recorded on a FM tape recorder (TEAC R-400), and the correlations were computed by a real time digital correlator (TEAC C-100) with the maximum delay time of 94 times the sampling period. The data were sampled at the periods of $0.4,1,2 \mathrm{msec}$ and the correlations were computed by the moving average method with the weight of $2^{-k}(k=10,11,12)$.

\section{Experimental Results}

Friction factor calculated from the time-averaged wall shear stress measured by the imbedded point electrode coincided with the Blasius empirical equation.

Velocity profile measured by a blunt-nose electrode was well represented by the universal velocity profile for each run.

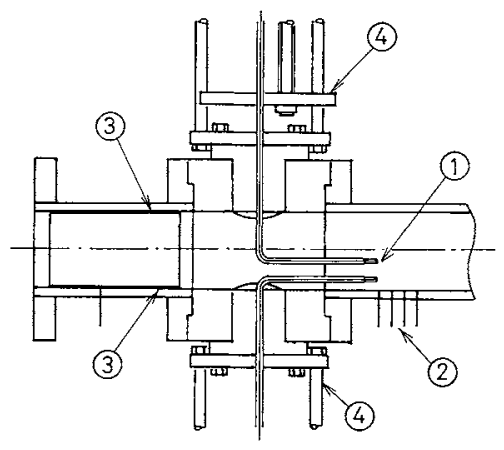

(1) Blunt-nose probe for measuring velocity

(2) Cathode for measuring shear stress at the wall

(3) Anode (Ni)

(4) Traverser

Fig. 1 Test section

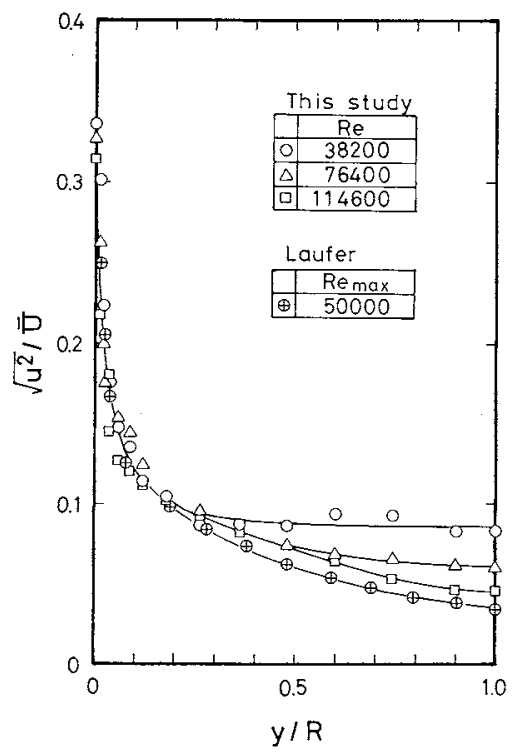

Fig. 2 Distributions of relative turbulence intensities of local axial velocity

Turbulent intensity distributions in the bulk region obtained from the current fluctuations of the bluntnose electrode are shown in Fig. 2, and compared with the data of Laufer ${ }^{7}$ at $R e_{\max }=50000$. Fairly good agreement can be seen.

Two point space-time correlations, i.e., $s-s$ correlation as a function of the separation $x$ and the delay time $\tau, s-u$ correlation as one of the distance $y$ and $\tau$, and $u-u$ correlation as one of the position of reference electrode $y_{r}$, the radial separation of electrodes $\Delta y$ and $\tau$ were measured respectively, these are defined below. A typical example of $s-u$ correlation is shown in Fig. 3, in which the delay time maximizing the correlation at a fixed $y$ gives the time lag $\tau_{y}$.

$s-s$ correlation between fluctuations of wall shear stress measured by two point electrodes with the longitudinal separation $x$, is defined as

$$
R_{s s}(x, \tau)=\frac{\left\langle s\left(x_{0}, t\right) \cdot s\left(x_{0}+x, t+\tau\right)\right\rangle}{\left[\left\langle s^{2}(x, t)\right\rangle \cdot\left\langle s^{2}\left(x_{0}+x, t\right)\right\rangle\right]^{1 / 2}}
$$




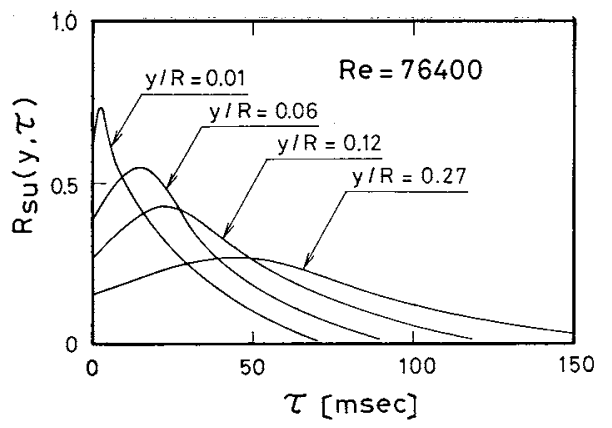

Fig. 3 Two point space-time correlations between fluctuations of wall shear stress and local velocity

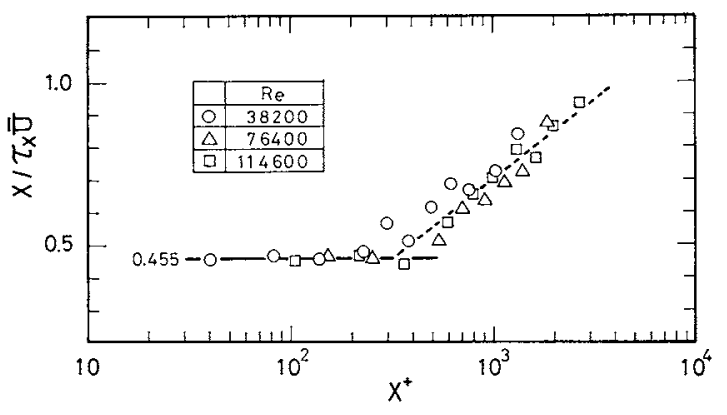

Fig. 4 Correlation of time lags $\tau_{x}$ in $s-s$ correlation

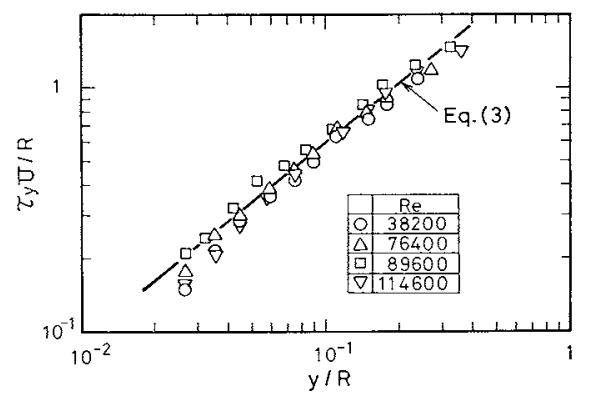

Fig. 5 Correlation of time lags $\tau_{y}$ in $s-u$ correlation

\begin{tabular}{lccc}
\hline $\begin{array}{l}\text { Table } 1 \text { Comparison of the disturbance velocities } \\
\text { the wall }\end{array}$ & \multicolumn{3}{c}{ near } \\
& $R e$ & $U_{s} / U$ & $U_{s}^{+}$ \\
\hline This work & 38,200 & 0.455 & 8.57 \\
(Local wall shear stress) & 74,600 & $\#$, & 9.33 \\
& 114,600 & $\#$ & 9.82 \\
Py (Local wall shear stress) & $3400-21500$ & & 6.3 \\
Hanratty & 15,900 & 0.058 & 0.978 \\
(Local mass transfer) & 21,000 & 0.062 & 1.125 \\
Liu (Streaming potential) & 75,000 & $0.7-0.8$ & \\
\end{tabular}

The $<>$ denotes the long time average of a statistically stationary quantity.

From the delay time $\tau_{x x}$ at which $R_{s s}$ at a fixed $x$ becomes maximum, the axial velocity of disturbance near the wall can be apparently obtained.

A plot of $x / \tau_{x} \bar{U}\left(=U_{s} / \bar{U}\right)$ vs. $x^{+}$gave a better correlation than $x / \tau_{x} u^{*}\left(=U_{s}^{+}\right)$vs. $x / A_{x}$ correlated by $\mathrm{Py}^{9}$, where $\bar{U}$ is the bulk mean velocity. However, it should be noted that though the ranges of $R e$ for these experiments were different, the correlation curves show the similar tendencies; that is, the disturbance velocity $U_{s}$ approaches a constant value as decreasing separation and $U_{s}$ increases as $x$ increases, as shown in Fig. 4.

In the present work, the constant value of $U_{s} / \bar{U}$ is obtained approximately as 0.455 for $x^{+}<330$.

Table 1 compares the available data on disturbance velocity. The results of this work agree with those of Py, and shows that the disturbance velocity corresponds to the local mean velocity at $y^{+} \fallingdotseq 9$. The rather large value of Liu's data ${ }^{8 !}$ may be due to the large separation of detectors. The too small values given by Hanratty ${ }^{10}$ are presumably caused by the difference between the measuring methods of shear stress and of mass transfer.

$s-u$ correlation between fluctuations of wall shear stress and local velocity at a distance $y$ from the wall in the same cross section $\left(x=x_{0}\right)$, is defined as

$$
R_{s u}(y, \tau)=\frac{\left\langle s\left(x_{0}, 0, t+\tau\right) \cdot u\left(x_{0}, y, t\right)\right\rangle}{\left[\left\langle s^{2}\left(x_{0}, 0, t\right)\right\rangle \cdot\left\langle u^{2}\left(x_{0}, y, t\right)\right\rangle\right]^{1 / 2}}
$$

From the measurements, it is found that fluctuation $s$ is always later than the corresponding fluctuation $u$, and the time lag $\tau_{y}$ maximizing $R_{s u}$ becomes larger with increasing $y$ from the wall. It has also been found that the correlation of $\tau_{y} U / R$ with $y / R$ is less scattered than the other correlations, such as $\tau_{y}^{+}$ vs. $y^{+}$presented by Eckelmann ${ }^{3)}$ and $\tau_{y}^{+} U^{+}$vs. $y^{+}$ etc., where $U$ is the local mean velocity at $y$. The correlation is shown in Fig. 5, and can be expresses by

$$
\tau_{y} U / R=3.7(y / R)^{0.8} \text { for } 0.03<y / R<0.4
$$

On the other hand, Eckelmann has given $\tau_{y}^{+}=y^{+}$for $y^{+}<5$.

$u-u$ correlation is defined as

$$
R_{u u}\left(y_{r}, \Delta y, \tau\right)=\frac{\left\langle u\left(x_{0}, y_{r}+\Delta y, t\right) \cdot u\left(x_{0}, y_{r}, t+\tau\right)\right\rangle}{\left[\left\langle u^{2}\left(x_{0}, y_{r}+\Delta y, t\right)\right\rangle \cdot\left\langle u^{2}\left(x_{0}, y_{r}, t\right)\right\rangle\right]^{1 / 2}}
$$

where $y_{r}$ denotes the position of the reference electrode, and $\Delta y$ is the radial distance between the traversed and the reference electrodes.

The signals from the traversed electrode $(\Delta y>0$, further from the wall than the reference one) always advance to the corresponding signals from the reference electrode. Denoting $\tau_{u}$ the time lag maximizing $R_{u u}$ at fixed $y_{r}$ and $\Delta y$ as same above, $\tau_{u}$ becomes a function of the position of electrodes and the flow characters at $y_{r}$ and $y_{r}+\Delta y$.

In the range of $\Delta y / R>0.05$, a proper correlation has been found in the relation $\left(\tau_{u} U / R\right)\left(y_{r} / R\right)^{0.2}$ vs. $(\Delta y / R)$ as shown in Fig. 6, which is expressed by

$$
\left(\tau_{u} U / R\right)\left(y_{r} / R\right)^{0.2}=5.71(\Delta y / R)^{1.66}
$$

It is noted that in this study the $u-u$ correlation has been measured for five reference positions, $0.117<$ 
$y_{r} / R<0.518, \Delta y / R<0.41$, at only one $R e$ of 89600 . In the range of $\Delta y / R<0.05$, the data scatter widely due to an uncertainty in measuring small values of $\tau_{u}$ and a small inevitable longitudinal separation between the tips of traversed and reference electrodes.

\section{Transmission Mechanism of Turbulent Disturbance}

Based on the experimental results that the fluctuating signals from the electrode at the farther distance from the wall always advance to the corresponding signals from the inner electrode as mentioned above, there is proposed a model concerning the transmission mechanism of turbulent disturbance.

Suppose an original disturbance at $x_{1}, y_{1}$ being convected in the flow direction at the local mean velocity $U_{1}$ according to the Taylor's hypothesis, and at the same time being transmitted in the radial direction by an eddy motion as shown in Fig. 7. Then, the time lag in the $s-u$ correlation is given by the difference between the time of the original disturbance reaching to the U-probe $\left(x=0, y_{1}\right)$ and the corresponding one arriving at the S-probe $(x=0, y=0)$. Because the path line of the eddy is given by

$$
d t=d x / U=d y / V_{t}
$$

then the time lag $\tau_{y}$ is expressed as

$$
\tau_{y}=\int_{0}^{y_{1}} \frac{1}{V_{t}} d y-\frac{1}{U_{1}} \int_{0}^{y_{1}} \frac{U}{V_{t}} d y=\int_{0}^{y_{1}} \frac{1}{V_{t}}\left(1-\frac{U}{U_{1}}\right) d y
$$

and also the time lag $\tau_{u}$ is similarly

$$
\tau_{u}=\int_{y_{r}}^{y_{1}} \frac{1}{V_{t}} d y-\frac{1}{U_{1}} \int_{y_{r}}^{y_{1}} \frac{U}{V_{t}} d y=\int_{y_{r}}^{y_{1}}-1-\left(1-\frac{U}{V_{t}}\right) d y
$$

Thus $\tau_{y}$ depends on $y_{1}$ and $\tau_{u}$ on $y_{r}$ and $y_{1}$ or $\Delta y$.

The transmission velocity $V_{t}$ is derived from Eq. (7) as

$$
\frac{1}{V_{t}}=-\frac{d}{d y} \frac{d \tau_{y} U / d y}{d U / d y}
$$

and from Eq. (8) as

$$
\frac{1}{V_{t}}=-\frac{d}{d y}-\frac{d \tau_{u} U / d y}{d U / d y}
$$

Since the position of U-probe, $y_{1}$, is arbitrary, the suffix " 1 " may be dropped out.

From the $s-u$ correlation, Eq. (3) leads to the expression for the transmission velocity in the $y$ direction by combining the universal velocity profile, $U^{+}=2.5 \ln y^{+}+5.5$, as follows

$$
V_{t}^{+}=1.06(y / R)^{0.2}
$$

Eckelmann $^{3 \prime}$ estimated the transmission velocity across the viscous sublayer based on the simple transmission model, which gave simply

$$
V_{s}^{+}=d y^{+} / d \tau_{y}^{+}=1
$$

This relation leads to $V_{t}^{+}=0.5$ according to the def-

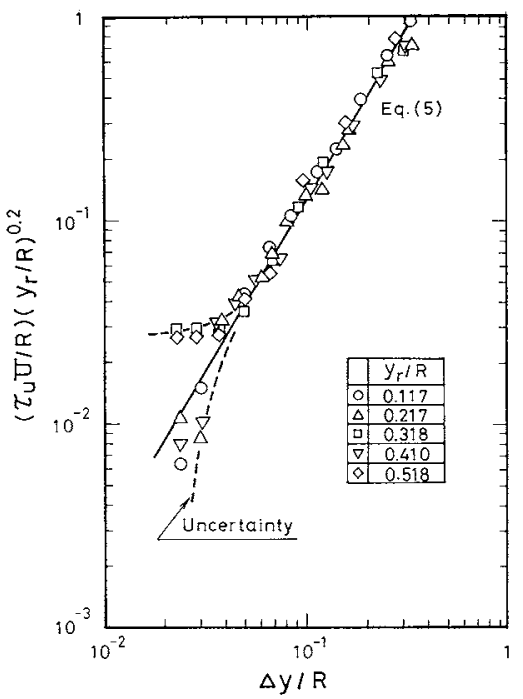

Fig. 6 Correlation of time lags $\tau_{u}$ in $u$ - $u$ correlation

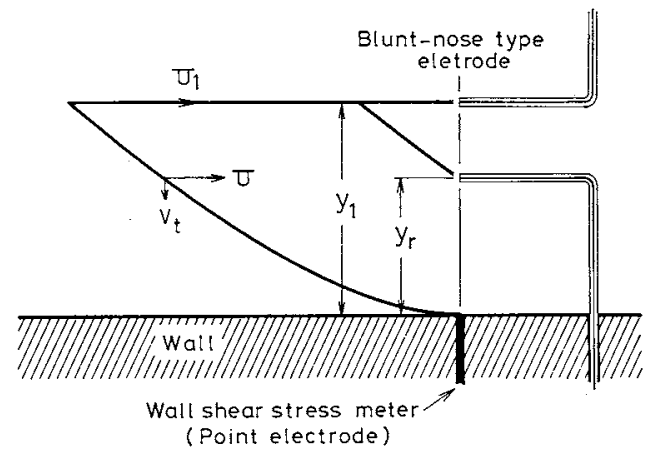

Fig. 7 Model of transmission of turbulent disturbance

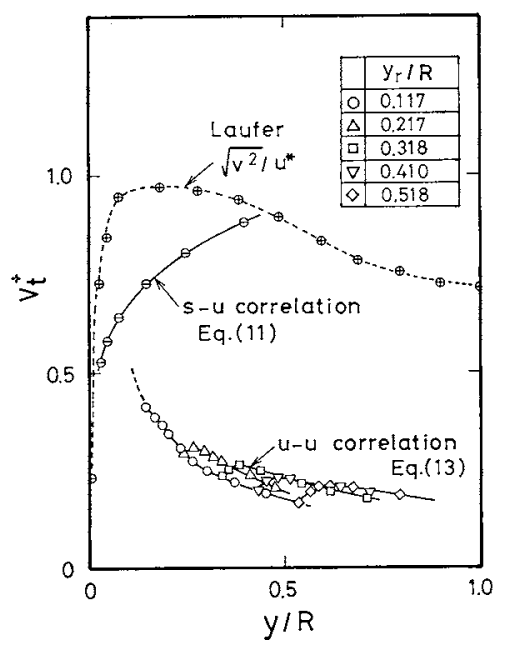

Fig. 8 Distribution of transmission velocities in the radial direction

inition in this study, Eq. (7).

From the $u-u$ correlations, the transmission velocity in the bulk region is derived from Eq. (5) as follows

$$
\begin{aligned}
V_{t}^{+}= & \left(y_{r} / R\right)^{0.2} / 3.79\left[(\Delta y / R)^{0.86}\right. \\
& \left.+0.66(y / R)(\Delta y / R)^{-0.34}\right]
\end{aligned}
$$

The transmission velocities $V_{t}^{+}$calculated by Eqs. (11) and (13) are shown in Fig. 8. $V_{t}^{+}$obtained from 


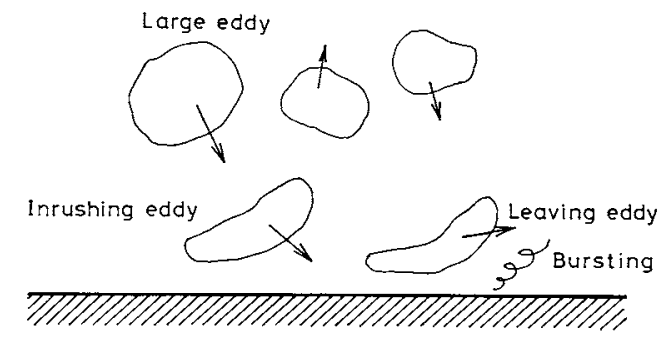

Fig. 9 Dynamic behavior of turbulent eddies

the $s-u$ correlation increases with increasing $y$, on the other hand $V_{t}^{+}$obtained from the $u-u$ correlation decreases on the whole while having a weak dependency on $y_{r}$. The opposite behaviors of transmission velocities which seem strange at first sight may be explained by introducing a dynamic process of eddies' motion based on the visual investigations ${ }^{2,6}$, which is shown in Fig. 9.

In the bulk region, eddies of relatively large scale move downstream while intermingling with each other. If an eddy has a sufficient radial velocity, it inrushes towards the wall and a part of which breaks down and creates a bursting eddy towards the center due to the strong interaction with the retarded flow near the wall. The remaining unbroken eddy is swept along the wall or by turning its direction slightly to the center by the following inrushing eddies, and then diffuses to the bulk region while intermingling with the eddies in that region.

Consider here the different behaviors between the transmission velocities $V_{t}$ from the $s-u$ and $u-u$ correlations in Fig. 8 with reference to the dynamic process mentioned above. The transmission velocity $V_{t}$ defined here may be considered corresponding to the statistical difference between the inward velocity $V_{i}$ and outward velocity $V_{o}$ of eddies, $V_{t}=V_{i}-V_{o}$.

In the $s-u$ correlation, the signals of inrushing, bursting and leaving eddies must have been measured. Of course, a proper conditional sampling of signals will give results corresponding to the bursting phenomena, that is the disturbance goes from the wall region to the bulk region $\left(V_{t}<0\right)$. However, from the results of $V_{t}>0$, it is considered that the inrushing disturbance has predominantly been observed for the long time intervals since the occurrence of bursting is very intermittent. Consequently, the transmission velocity $V_{t}$ corresponds mainly to the inrushing velocity, and it decreases towards the wall due to the wall effect.

In the $u-u$ correlation, the reference electrode nearest the wall was set at $y_{r} / R=0.117$ or $y^{+}=250$, where the strong bursting phenomena might have finished according to the visual study by Corino et $\mathrm{al}^{2}{ }^{2}$. In that case the U-probes would have measured mainly the signals of inward and outward eddies.
As mentioned above, the leaving eddy increases its velocity towards the center with intermingling with the eddies in the bulk region. Consequently the transmission velocity $V_{t}=V_{i}-V_{o}$ decreases towards the center.

In Fig. 8, the transmission velocities are also compared with the intensity of radial component of velocity $v$ measured by Laufer ${ }^{7}$. The comparison of these quantities may have less meaning, since the transmission velocity $V_{t}$ from the $s-u$ correlation corresponding to the inrush velocity is Lagrangian but the intensity $v$ is Eulerian. However, it is interesting that these values are of the same order of magnitude.

\section{Summarising Remarks}

A two point space-time correlation technique was applied to measuring $R_{s s}(x, \tau), R_{s u}(y, \tau), R_{u u}\left(y_{r}, \Delta y, \tau\right)$ in fully developed turbulent pipe flow.

The representation for the time lags $\tau_{x}, \tau_{y}$ and $\tau_{u}$, better than the previous reported ones, have been shown in Fig. 4, 5 and 6, and the correlating equations, Eq. (3) for $\tau_{y}$ and Eq. (5) for $\tau_{u}$ have been obtained.

The variation of the time lag $\tau_{x}$ in the flow direction revealed the disturbance velocity $U_{s}$ along the wall as being proportional to the cross sectional average velocity $\bar{U}$.

Based on the variations of time lags $\tau_{y}$ and $\tau_{u}$ in the radial direction, the transmission model of turbulent disturbance has been proposed on which the transmission velocities have been derived as Eqs. (11) and (13). The transmission velocity from the $s-u$ correlation, Eq. (11), is proportional to $(y / R)^{0.2}$ and corresponds to the velocity of inrushing eddy. On the other hand, Eq. (13) from the $u-u$ correlation gives the transmission velocity in the bulk region, which decreases towards the center. This opposite behavior shows the further importance of measuring the $u-u$ correlations in the wall region. The velocity of inrushing eddy is the same order as the intensities of radial velocity component, though those are Lagrangian but these are Eulerian.

In this study, the transmission mechanism of turbulent disturbance has been discussed only from the time lags maximizing the correlation functions. To understand fully the Lagrangian behavior of turbulent eddies, it is necessary to measure additional correlations such as $R_{u u}(x, y, \tau)$ and discuss the values of correlation functions in addition to the time lags.

\section{Nomenclature}

$\begin{array}{llr}D & =\text { diameter of pipe } & {[\mathrm{cm}]} \\ L & =\text { length from entrance } & {[\mathrm{cm}]} \\ R & =\text { radius of pipe } & {[\mathrm{cm}]} \\ R e & =\text { Reynolds number }=2 \rho R \bar{U} / \mu & {[-]} \\ R e_{\max } & =2 \rho R U_{\max } / \mu & {[-]}\end{array}$




\begin{tabular}{|c|c|c|c|}
\hline$R_{s s}$ & \multicolumn{3}{|c|}{$=$ correlation between fluctuations of wall } \\
\hline$R_{s u}$ & $=$ & $\begin{array}{l}\text { correlation between fluctuations of wall } \\
\text { shear stress and local velocity, defined b } \\
\text { Eq. (2) }\end{array}$ & by \\
\hline$R_{u u}$ & $=$ & $\begin{array}{l}\text { correlation between fuctuations of local } \\
\text { velocities, defined by Eq. (4) }\end{array}$ & {$[-]$} \\
\hline$s$ & $=$ & fluctuation of wall shear stress & $\left.\mathrm{g} / \mathrm{cm} \cdot \mathrm{sec}^{2}\right]$ \\
\hline$t$ & $=$ & time & [sec] \\
\hline$U$ & $=$ & local mean velocity & {$[\mathrm{cm} / \mathrm{sec}]$} \\
\hline$\vec{U}$ & $=$ & cross sectional average velocity & {$[\mathrm{cm} / \mathrm{sec}]$} \\
\hline$U_{\max }$ & $=$ & maximum velocity & {$[\mathrm{cm} / \mathrm{sec}]$} \\
\hline$U_{s}$ & & $\begin{array}{l}\text { disturbance velocity of eddy along } \\
\text { the wall }\end{array}$ & {$[\mathrm{cm} / \mathrm{sec}]$} \\
\hline$u$ & $=$ & fluctuation of local axial velocity & {$[\mathrm{cm} / \mathrm{sec}]$} \\
\hline$u^{*}$ & $=$ & friction velocity & {$[\mathrm{cm} / \mathrm{sec}]$} \\
\hline$V_{i}$ & $=$ & inward velocity of eddy to the wall & {$[\mathrm{cm} / \mathrm{sec}]$} \\
\hline$V_{o}$ & $=$ & outward velocity of eddy from the wall & $11[\mathrm{~cm} / \mathrm{sec}]$ \\
\hline$V_{s}$ & & $\begin{array}{l}\text { transmission velocity of turbulent } \\
\text { disturbance in the radial direction, } \\
\text { defined by Eqs. (12) }\end{array}$ & {$[\mathrm{cm} / \mathrm{sec}]$} \\
\hline$V_{t}$ & & $\begin{array}{l}\text { transmission velocity of turbulent } \\
\text { disturbance in the radial direction, } \\
\text { defined by Eq. (6) }\end{array}$ & {$[\mathrm{cm} / \mathrm{sec}]$} \\
\hline$v$ & $=$ & fluctuation of local radial velocity & {$[\mathrm{cm} / \mathrm{sec}]$} \\
\hline$x$ & $=$ & Iongitudinal distance & [cm] \\
\hline$x^{+}$ & $=$ & $\rho x u^{*} / \mu$ & {$[-]$} \\
\hline$y$ & $=$ & radial distance from the wall & [cm] \\
\hline$y_{r}$ & $=$ & distance of reference probe from the wa & [cm] \\
\hline$y^{+}$ & $=$ & $\rho y u^{*} / \mu$ & {$[-]$} \\
\hline
\end{tabular}

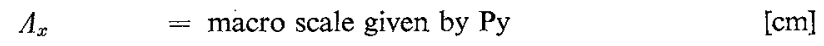
$\rho \quad=$ density $\quad\left[\mathrm{g} / \mathrm{cm}^{3}\right]$
$\mu \quad=$ viscosity $\quad[\mathrm{g} / \mathrm{cm} \cdot \mathrm{sec}]$
$\tau \quad=$ time lag [sec]
$\tau_{x} \quad=$ time lag maximizing $R_{s s} \quad$ [sec]
$\tau_{y} \quad=$ time lag maximizing $R_{s u} \quad$ [sec]
$\tau_{u} \quad=$ time lag maximizing $R_{u u} \quad$ [sec]
$\tau^{*} \quad=\tau u^{* 2 / \nu} \quad[-]$

\section{Literature Cited}

1) Blackwelder, R. F. and L. S. G. Kovasznay: Phys. Fluids, 15, 1545 (1972).

2) Corino, E. R. and R. S. Brodkey: J. Fluid Mech., 37, 1 (1969).

3) Eckelmann, H: Bericht Nr. 101/1973., Max-Plank Inst, Strom. u. Aerodyn. Versuch, Göttingen (1973).

4) Favre, A., J. Gaviglio and R. Dumas: Phys. Fluids, 10, S138 (1967).

5) Jones, B. G., H. P. Planchon and R. J. Hammersley: AIAA $J ., 11,1146$ (1973).

6) Kim H. T., S. J. Kline and W. C. Reynolds: J. Fluid Mech., 50, 133 (1971).

7) Laufer, L: NACA TR 1174 (1954).

8) Liu, H: AlChE J., 13, 644 (1967).

9) Py, B.: Int. J. Heat Mass Transfer, 16, 145 (1973).

10) Shaw, P. V. and T. J. Hanratty: AIChE J. 10, 475 (1964).

11) Shlien, D. J. and S. Corrsin: J. Fluid Mech., 62, 255 (1974).

(Presented in part at the 36th Annual Meeting, Tokyo (1971) and the 11th General Symposium, Kyoto (1973), of The Soc. of Chem. Engrs., Japan.) 